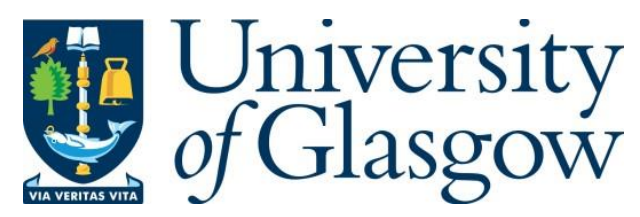

Coia, M. and Hammond, G. (2016) Self assessment: acute-onset vomiting in a Tibetan Terrier. Companion Animal, 21(1), pp. 38-42.

There may be differences between this version and the published version. You are advised to consult the publisher's version if you wish to cite from it.

http://eprints.gla.ac.uk/154525/

Deposited on: 8 February 2018

Enlighten - Research publications by members of the University of Glasgow

http://eprints.gla.ac.uk 
1 Canine Imaging Self Assessment: Acute onset vomiting in a Tibetan Terrier

3 Signalment and History

5 A 3-month-old female entire Tibetan Terrier was presented for investigation of acute

6 onset vomiting and lethargy. The patient had experienced vomiting for 48 hours,

7 which was also associated with an episode of diarrhoea. The owner had noted poor

8 body growth, poor weight-gain and suboptimal body condition despite demonstration

9 of a good appetite prior to her presenting complaint. She had no associated clinical

10 signs of polydipsia or polyuria at home. To the owner's knowledge there has been no

11 access to any known toxin, or evidence of ingesting foreign material that could result

12 in gastrointestinal obstruction, but halitosis had been noted for approximately 8

13 weeks. She was fully vaccinated and had been treated routinely for endo- and 14 ectoparasites.

\section{Clinical examination}

17 On presentation, the dog was lethargic but was responsive with a normal body 18 temperature. There was an increased respiratory rate (60 breaths per minute) without 19 dyspnoea. The mucous membranes were pink, without evidence of congestion and 20 with a capillary refill time of <2seconds. The heart rate was 180 beats per minute 21 with normal pulse quality. On auscultation of the thoracic cavity, the lungs 22 demonstrated increased respiratory noises with pulmonary crackles. Abdominal 23 examination was unremarkable. 
25 Survey radiographs of thorax and abdominal were obtained in right lateral recumbency to screen for causes of acute vomiting (Figure 1).

Describe the main findings on the radiograph. Based on the findings construct a differential diagnosis list.

Figure 1 is a right lateral projection of the thorax and abdomen. There are thin, linear, radio-opacities that appear to be contained within the gastric wall - these are suggestive of mineralisation of the rugae. There is a mild reduction of the peritoneal serosal detail in the patient - this can be commonly observed in a young patient due to the brown fat deposits within the abdomen. The peritoneal definition can be altered in patients with free abdominal fluid - there is no evidence of this in this case. The vertebrae and both femurs demonstrate decreased bone opacity radiographically, and there is thinning of the cortices of the distal portion of the femur giving a double cortical sign seen with osteopenia; this is due to intracortical bone resorption seen with cases of nutritional secondary hyperparathyroidism, suspected renal secondary hyperparathyroidism. There is evidence of loss of the fine trabeculation of the vertebral bodies, spinous processes and the distal portion of both femurs, leaving a coarse pattern and generalised loss of bone density. On assessment of the thoracic cavity, there is a diffuse opacity affecting the lung fields; this appears to be an interstitial lung pattern. The thoracic findings are likely to normal for age of the dog; this is thought to be of no clinical significance and was not further investigated.

\section{Key radiographic findings:}

- Mineralisation within the stomach, suspected gastric mucosal mineralisation. 
51 Differential diagnosis:

52 - Primary Hyperparathyroidism

53 - Secondary Renal hyperparathyroidism

54 - Uraemia (uraemic gastropathy) secondary to renal failure may be suspected 55 due to the tissue mineralisation of the gastric wall.

56 - Gastric foreign body (chronic) - this was thought to be less likely but a 57 radio-dense foreign body may be considered.

59 What other investigations should be considered?

60 In this case, with the clinical manifestations, signalment, and the radiographic 61 findings, there was a suspicion of chronic renal disease (secondary dystrophic 62 calcification of the gastric wall with radiographic evidence of osteopenia). Due to the 63 systemic signs noted routine biochemistry and haematology were performed. The 64 results of the bloods are shown in Table 1 (haematology) and Table 2 (serum 65 biochemistry).

66

67 The haematology revealed a mild anaemia that appeared poorly regenerative in nature 68 (normocytic (normal mean cell volume [MCV]), normochromic (normal mean cell 69 haemoglobin concentration $[\mathrm{MCHC}]$ ) and low to normal reticulocyte count). The 70 biochemistry results showed a marked elevation in the serum urea (BUN) and 71 creatinine levels. There was hyponatraemia and hypocholaemia noted. The 72 electrolytes abnormalities could be associated with excessive gastrointestinal losses 73 (including vomiting $\mathrm{Cl}$ - rich stomach contents) or through excessive urinary losses. 
Potential causes of azotaemia:

1. Pre-renal Azotemia: In this circumstance there is reduced renal perfusion due

to low blood volume. There may be accelerated production of nitrogenous waste products (e.g. BUN) because of enhanced catabolism of tissues in association with infection, fever, trauma, or the use of corticosteroids . BUN can become elevated with gastrointestinal digestion and absorption of protein sources- this can include a high-protein diet or gastrointestinal hemorrhage.

2. Renal Azotemia: Acute or chronic kidney conditions that impair at least seventy-five per cent of the renal function can result in an ensuing azotaemia.

3. Post-renal Azotemia: The azotaemia is usually the result of altered urine excretion such as urinary obstruction or rupture of the urinary tract. Urinary clearance and excretion of the nitrogenous products is altered.

87

Additional ancillary tests that would be indicated include urinalysis, blood gas analysis, serum phosphate, and serum calcium levels (total and ionised), to further identify the underlying pathology. Urinalysis acts as a basic, inexpensive and quick diagnostic tool, and can evaluate for renal and non-renal causes of azotaemia; urine specific gravity can facilitate the assessment of kidney function and concentrating ability of solutes.

Which imaging modality would you use next and what would you be looking for? Abdominal ultrasonography was performed to assess the renal architecture and shape, and allow further assessment of the gastrointestinal tract. Still ultrasonographic images are shown of the stomach (Figure 2a), left kidney (Figure 2b) and right kidney

99 (Figure 2c). Additional to the abdominal imaging, a dorsoventral radiograph was 
taken of the patient's skull (Figure 3).

101

What are the major abnormalities on the sonographic images? What additional information is available from the radiograph of the skull?

The stomach wall (Figure 2a) appears to be thickened, with hyperechoic mucosa. The images are consistent with inflammation and mineralisation affecting the stomach

107 wall - this is most likely due to uraemic gastritis. There was no suggestion of a 108 gastrointestinal foreign body on assessment of the gastrointestinal tract; there was an 109 empty stomach without shadowing from a foreign interface, while the small intestines 110 were within normal limits without a gravel sign.

111 The ultrasound images of both kidneys (Figures $2 \mathrm{~b}$ and $2 \mathrm{c}$ ) show a generalised 112 increase in echogenicity and a loss of the normal renal architecture; there is poor 113 corticomedullary differentiation. The kidney parenchyma is difficult to identify when 114 compared to the surrounding tissue, and these changes indicate chronic or dysplastic 115 renal changes. The kidneys were not measured at the time of the abdominal 116 examination - this may be useful as dysplastic kidneys are usually smaller on 117 ultrasonography.

118 The skull radiograph (Figure 3) was taken to assess for loss of the lamina dura. There 119 is loss of the lamina dura surrounding the alveolus. There is generalised reduction in 120 the bone density, with loss of alveolar bone and ill defined lucency around the teeth of 121 the maxilla and mandibular dental arcades (the "floating teeth" appearance) giving a 122 generalised, coarse, lace like trabecular bone pattern. The cortices appear irregular123 this is most marked at the zygomatic arch and the coronoid process. The radiographic 124 changes are suggestive of secondary renal hyperparathyroidism; the loss of the lamina 
125 dura is an early radiographic sign, followed by generalised loss of bone density of the 126 skull and long bones.

128 What is the presumed diagnosis for this case?

129 The young age combined with the clinical findings on bloods and imaging is 130 suggestive of a juvenile nephropathy; the most likely diagnosis is renal dysplasia 131 resulting in progressive chronic renal failure, with secondary renal 132 hyperparathyroidism and uraemic gastritis.

\section{Discussion and outcome.}

135 Renal dysplasia (RD) should be considered as a differential in juvenile patients with 136 renal disease that exhibit an appropriate history, signalment and clinical findings, 137 especially patients that have had no exposure to toxin ingestion. RD is thought to 138 have a strong familial link with certain pure-breeds showing a predisposition, 139 including but not limited to Boxers, Cocker Spaniels, Chow Chow, Golden Retriever, 140 Lhasa Apso, Samoyed, Shih Tzu and Soft Coated Wheaten Terrier. It is a congenital 141 and developmental malformation of the kidneys, which results from poor 142 differentiation of the renal tissue, with the progressive manifestations associated with 143 chronic kidney disease. The age of onset of clinical signs in congenital nephropathies 144 ranges from a few weeks to several years of age, however many can remain quiescent 145 until later in life. The clinical manifestations can vary in each patient, with the most 146 common presenting signs being a reduced appetite, stunted growth or weight loss, 147 polyuria and polydipsia, and varying gastroenteric signs.

148 Often affected dogs appear healthy and exhibit good urine concentrating ability 149 initially. Proteinuria often develops followed by a reduced growth rate and reduced 
urine concentrating ability. Subsequently serum concentrations of urea and creatinine

151 progressively increase. Additionally, such patients will have other clinical findings

152 associated with the chronic renal insufficiency: non-regenerative anaemia, azotaemia,

153 hyperphosphataemia, hypokalaemia and a metabolic acidosis. Calcium levels are

154 variable in these patients and may demonstrate low (may relate to low albumin 155 levels), normal or high levels depending on the stage of the disease. The urine specific 156 gravity is commonly isosthenuric $(1.008-1.012)$ in such patients despite the animal 157 showing azotaemia.

158

159 Ultrasound proves to be an effective tool to assess the kidney structure, giving 160 supportive evidence for the clinical suspicions and facilitating the formulation of a 161 concise differentials list based on the imaging findings. The ultrasound findings of 162 renal dysplasia have been documented in dogs. The ultrasound appearance of 163 affected kidneys may reveal thinning of the renal cortex with increased echogenicity 164 or poor corticomedullary definition when compared to normal structure, with diffuse 165 increased echogenicity of the parenchyma. There may be notable distortion affecting 166 the renal pelves in affected patients and the kidneys are usually bilaterally reduced in 167 size. Ultrasound alone cannot differentiate RD from other causes of fibrotic, end stage 168 kidneys, although the age of the patient and other historical findings will usually 169 indicate the likely cause.

171 When uraemia occurs, a degenerative, ulcerative gastritis can occur with 172 consequential mineralization of the gastric mucosa and lamina propria (uraemic 173 gastropathy) as seen in this case. Calcium homeostasis becomes altered in many 174 patients suffering from renal failure due to acidosis, early hyperphosphatemia, 
secondary parathyroid hyperplasia and poor synthesis of 1,25

176 dihydroxycholecalciferol by the diseased kidneys. Calcification of the tissues appears

177 to be due to a combination of ischaemia, tissue degeneration and altered plasma

178 concentrations of calcium and phosphorus.

179 Renal dysplasia cannot be definitively diagnosed from imaging or bloods alone, but 180 requires histological examination of the renal tissues following biopsy or at necropsy 181 (although the presentation and sonographic findings will often raise a very high index 182 of suspicion).

183 There is no specific treatment for this condition and in clinically affected dogs 184 showing with polydipsia, polyuria and uraemia, it continues to end-stage renal 185 disease. The prognosis in this condition is very poor. These animals often decline 186 rapidly despite supportive treatment; the poor quality of life often results in 187 euthanasia. This dog was sadly euthanised due to advancement of the progressive 188 clinical manifestations.

\section{Further Reading}

191 Abraham LA, Beck C, Slocombe RF. Renal dysplasia and urinary tract infection in a 192 Bull Mastiff puppy. Aust Vet J. 2003;81: 336-339.

193 Barr F (2006). Long Bones - Juvenile. In: BSAVA Manual of Canine and Feline

194 Musculoskeletal Imaging (Barr FJ, Kirberger RM eds.), British Small Animal

195 Veterinary Association, Gloucester, pp 19-31.

196 Cheville NF. Uremic gastropathy in the dog. Vet Pathol. 1979;16: 292-309.

197 Cowgill LD, Francey T (2005) Acute Uremia In: Ettinger SJ, Feldman EC, eds.

198 Textbook of Veterinary Internal Medicine $6^{\text {th }}$ Edition. St. Louis, Elsevier Saunders: .

199 pp. $1731-1751$ 
200 Graham JP (2011). Kidneys and Proximal Ureters. In: BSAVA Manual of Canine

201 and Feline Ultrasonography, Barr F, Gaschen L eds. British Small Animal Veterinary

202 Association, Gloucester, pp 110-123.

203 Grooters AM, Miyabayashi MST, Biller DS, Merryman J. Sonographic appearance of

204 uremic gastropathy in four dogs. Vet Radiol Ultrasound. 1994;35: 35-40.

205 Hoppe A, Swenson L, Jönsson L, Hedhammar A. Progressive nephropathy due to 206 renal dysplasia in shih tzu dogs in Sweden: A clinical pathological and genetic study.

207 J Small Anim Pract. 1990;31: 83-91.

208 Lees GE, Helman RG, Kashtan CE, Michael AF, Homco LD, Millichamp NJ, et al. A 209 model of autosomal recessive Alport syndrome in English cocker spaniel dogs.

210 Kidney Int. 1998;54: 706-719.

211 Peeters D, Clercx C, Michiels L, Desmecht D, Snaps F, Henroteaux M, et al. Juvenile 212 nephropathy in a boxer, a rottweiler, a collie and an Irish wolfhound. Aust Vet J. 213 2000;78: 162-165.

214 Peters RM, Goldstein RE, Erb HN, Njaa BL. Histopathologic features of canine 215 uremic gastropathy: a retrospective study. J Vet Intern Med. 2005;19: 315-320.

216 Polzin DJ, Osborne CA, Ross A (2005) Chronic Kidney Disease In: Ettinger SJ,

217 Feldman EC, eds. Textbook of Veterinary Internal Medicine $6^{\text {th }}$ Edition. St. Louis,

218 Elsevier Saunders: pp. $1756-1785$

219 Seiler G, Mai W (2012). The Stomach. In: BSAVA Manual of Canine and Feline 220 Abdominal Imaging (O’Brien R, Barr F eds.), British Small Animal Veterinary

221 Association, Gloucester, pp 87-109.

222 Figure Legends

223

224 Figure 1: Right Lateral Recumbency radiograph of the caudal thorax and abdomen. 
225 Figure 2a: Long-axis ultrasonogram of the body of the stomach

226 Figure 2b: Long-axis ultrasonogram of the left kidney

227 Figure 2c: Long axis ultrasonogram of the right kidney

228 Figure 3: Dorsoventral radiograph of the skull.

229

230

231

232

233

Tables:

Table 1: Haematology

\begin{tabular}{ccc} 
Parameter & Value & Reference Range \\
RBC (x1012/l) & $\mathbf{4 . 2}$ & $5.65-8.87$ \\
HCT (\%) & $\mathbf{2 5 . 8}$ & $37.3-61.7$ \\
Hb (g/dl) & $\mathbf{9 . 7}$ & $13.2-20.5$ \\
MCV (\%) & $\mathbf{6 1 . 4}$ & $61.6-73.5$ \\
MCHC (\%) & 37.6 & $32-37.9$ \\
Reticulocytes (K/uL) & 38.6 & $10-110$ \\
Platelets (K/ul) & 241 & $148-484$ \\
WBC (x109/l) & 8.88 & $5.05-16.76$ \\
Neutrophils (x109/1) & 5.46 & $2.95-11.64$ \\
Lymphocytes (x109/l) & 2.75 & $1.05-5.10$ \\
Monocytes (x109/l) & 0.43 & $0.16-1.12$ \\
Eosinophils (x109/1) & 0.24 & $0.06-1.23$ \\
Basophils (x109/l) & 0 & $0-0.1$ \\
\hline
\end{tabular}


Abnormalities are highlighted in bold

235

Table 2: Serum biochemistry

$\begin{array}{ccc}\text { Parameter } & \text { Value } & \text { Reference Range } \\ \text { Glu (mmol/l) } & 6.12 & 4.28-8.34 \\ \text { BUN/ Urea (mmol/l) } & \mathbf{4 6 . 4} & 2.5-10.4 \\ \text { Creat (mmol/l) } & \mathbf{2 8 9} & 27-106 \\ \text { TP (g/L) } & 54 & 48-72 \\ \text { Alb (g/L) } & 28 & 21-36 \\ \text { Glob (g/L) } & 26 & 23-38 \\ \text { Alb:Glob } & & 8-75 \\ \text { ALT (U/l) } & \mathbf{1 5 5} & 46-337 \\ \text { ALKP (U/l) } & 121 & 145-157 \\ \text { Na( mmol/l) } & \mathbf{1 3 8} & 35-5.5 \\ \text { K (mmol/l) } & 5.2 & 105-119 \\ \text { Cl (mmol/l) } & \mathbf{9 8} & \end{array}$

